\title{
Mesoscale flow and heat transfer modelling and its application to liquid and gas flows
}

\author{
Nikolaos Asproulis, Marco Kalweit, Evgeniy Shapiro and \\ Dimitris Drikakis
}

\author{
Cranfield University, United Kingdom \\ Aerospace Sciences Department \\ Fluid Mechanics \& Computational Science Group \\ d.drikakis@cranfield.ac.uk
}

\begin{abstract}
Advances in micro and nanofluidics have influenced technological developments in several areas, including materials, chemistry, electronics and bio-medicine. The phenomena observed at micro and nanoscale are characterised by their inherent multiscale nature. Accurate numerical modelling of these phenomena is the cornerstone for enhancing the applicability of micro and nanofluidics in the industrial environment. We investigated different strategies for applying macroscopic boundary conditions to microscopic simulations. Continuous rescaling of atomic velocities and velocity distribution functions, such as Maxwell-Boltzmann or ChapmanEnskog, were investigated. Simulations were performed for problems involving liquids and gases under different velocity and temperature conditions. The results revealed that the selection of the most suitable method is not a trivial issue and depends on the nature of the problem, availability of computational resource and accuracy requirement.
\end{abstract}

Keywords: multiscale modelling, heat transfer, molecular dynamics, hybrid atomistic continuum methods, micro/nanofluid dynamics

\section{INTRODUCTION}

During the last decade, nanofluidics has emerged as a strongly emerging field [1] and has already found applications in various disciplines including bioengineering, medicine and chemistry [2]. In the near future, it is also expected to have impact in many areas, such as drug delivery and nano-manufacturing.

Nanoscale devices have unique characteristics and present significant differences compared to larger scale ones. Surface properties for this type of devices are dominant and, therefore, understanding of the phenomena involved, as well as efficient and realistic modelling of these phenomena is of paramount importance [3]. Traditional continuum flow models, based on Navier-Stokes equations with no-slip boundary conditions are not applicable for microscale systems, such as combustors, valves, nozzles and turbomachines [3], due to the break down of the continuum approximation. In particular, the macroscopic constitutive relations and boundary conditions become inadequate [4]. This discrepancy increases further for nanofluidic devices, where molecular modelling must be employed. Molecular simulations are computationally intensive, thereby restricting their application to nanoscale systems and time scales below microseconds. Thus, modelling nanofluidic devices presents a significant challenge due to the inaccuracy of the continuum models and inefficiency of the molecular ones in the meso scale regimes. To address this challenge, multiscale methods that bridge the gap between microscopic and macroscopic description have been proposed [5]. One group of multiscale methods for nano- and microscale systems couple molecular and continuum solvers to provide a unified description from nanoscale to larger scales and are called hybrid methods. 
A number of hybrid methods for fluidics have been proposed in the literature [5-15].The majority of these methods are based on the domain decomposition technique which is suitable for problems where the continuum equations are valid in most parts of the computational domain, but fail to fully describe the phenomena in a particular area. The main idea is to decompose the simulation domain into two distinct regions. One region is modelled as a continuum and solved by a continuum fluid dynamics (CFD) solver, while the other region is modelled using molecular dynamics (MD) [5,6]. Within each region the solution is separately computed and both regions are linked by a hybrid solution interface (HSI). The advantage is that the computationally expensive MD solver is only applied within a small region, whereas the majority of the simulation domain is solved by a CFD solver, which is several orders of magnitude faster $[6,16]$. The domain decomposition technique does not decouple the time scales between the macroscopic and microscopic scales. Thus, the maximum computational time is restricted to time scales computed by MD. In order to handle the time scale constraints a pointwise coupling (PWC) method has been recently proposed [13,17]. In the PWC, the entire domain is computed by the macroscopic solver while the microscale model enters as a refinement and is used locally for obtaining macroscopic properties from the microscopic model. One important property of the PWC is that it naturally decouples the microscopic and macroscopic timescales.

The accuracy and efficiency of multiscale approaches depend to a great extent on the boundary condition transfer (BCT) method that constrains the atomistic region to the continuum conditions. The problem of imposing macroscopic conditions on a molecular system is a very challenging task and has not yet been addressed for a general case [16,18]. The main difficulty is the disparity between degrees of freedom modelled by the atomistic and continuum models.

This paper investigates mesoscale methods for applying macroscopic boundary conditions to a microscopic system, in particular temperature enforcement. However, the anticipated impact of this work is broader as these techniques can be implemented to other multiscale problems. In the simulation problems presented in this paper, MD is used within the PWC for obtaining macroscopic properties either near the boundary of the continuum domain or at specific points inside the flow domain. The paper is organised as follows. Sec. 2 provides a brief description of MD, the PWC coupling scheme and the methods for enforcing the continuum states in the MD domain. The results for liquid flow cases are presented in Sec. 3.1 and Sec. 3.2. In Sec. 3.3 the results of gaseous flow simulations are discussed. The conclusions are summarised in Sec. 4.

\section{METHODOLOGY}

\subsection{Molecular Dynamics}

In the PWC method, MD simulations are used to calculate macroscopic properties of the fluid. The nature of MD method is deterministic so that if velocities, positions and equations of motions of the components of a molecular system are known at time $t$, the state of the systems is fully determined at time $t+\Delta t$. Atoms are modelled as mass points with position $\boldsymbol{r}_{i}$ and mass $m_{i}$. Their motion is governed by Newton's equation of motion, written for each atom $i$ in the following form

$$
m_{i} \ddot{\boldsymbol{r}}_{i}=-\frac{\partial V_{i}}{\partial \boldsymbol{r}_{i}}
$$

The potential energy $V_{i}$ is the sum of semi-empirical analytical functions that represent the interatomic forces. The atomic trajectories are calculated by integrating Eq. (1) in time for all atoms. Despite the apparent simplicity, the simulations are computationally demanding due to the large number of atoms involved. Nowadays, modern parallel computers allow MD simulations of several millions of atoms [19]. The MD simulations in this study were performed using a modified version of LAMMPS [20], which employs the velocity Verlet scheme [21] to integrate Eq. (1). 


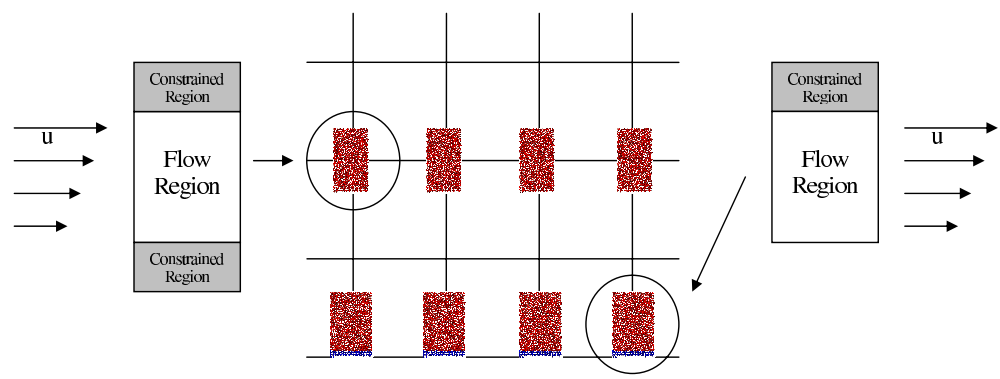

Fig. 1. Schematic representation of PWC.

All interatomic interactions are modelled by the shifted Lennard-Jones (LJ) 6-12 potential. Between a pair of atoms $i$ and $j$ with the distance $r_{i j}$, the LJ potential is given by

$$
V_{i j}^{L J}=4 \epsilon\left[\left(\frac{\sigma}{r_{i j}}\right)^{12}-\left(\frac{\sigma}{r_{i j}}\right)^{6}-\left(\frac{\sigma}{r_{c}}\right)^{12}+\left(\frac{\sigma}{r_{c}}\right)^{6}\right] .
$$

The cut-off distance is set to $r_{c}=2.5 \sigma$.

Depending on the choice of $\epsilon, \sigma$ and the mass $m$, the LJ-potential can represent a number of elements whose atoms interact via Van-der-Waals forces. Unless otherwise stated, reduced units are used throughout the paper. For Argon these would represent $\epsilon=\epsilon_{A r}=119.8 \mathrm{~K} / \mathrm{k}_{\mathrm{B}}, \sigma=$ $\sigma_{A r}=0.34 \mathrm{~nm}, m=m_{A r}=0.34 \mathrm{amu}$. The corresponding time unit is $\tau=\left(\mathrm{m} \sigma^{2} / \epsilon\right)^{1 / 2}=$ $2.16 \mathrm{ps}$.

\subsection{Pointwise coupling scheme}

In the PWC scheme, the CFD solver advances the solution in the entire domain. MD simulations are used to (i) provide accurate boundary conditions, such as slip velocity, tangential stress or heat flux; (ii) substitute the constitutive relations, for example, to calculate pressure $(P(\rho, T))$; and (iii) calculate transport properties, such as viscosity for non-Newtonian fluids. In the most simplistic PWC scheme the MD simulations are performed at every CFD time step, corresponding to the local current continuum states. The macroscopic quantities of interest are measured from the MD simulations and fed back to the CFD solver, where they are used to advance the solution. Obviously, this procedure leads to repetitive MD simulations of nearly identical states and, thus, a more sophisticated algorithm that stores and makes use of already performed MD simulations may be employed instead.

In order to perform MD simulations for the required continuum states, they must be constrained in an appropriate manner. Initially, this is achieved by setting up the molecular system with the corresponding continuum density $\rho_{\text {con }}$ and temperature $T_{\text {con }}$. During the actual MD simulations, the molecular system is constrained to the continuum velocity gradient and temperature by controlling the molecular state within confined regions on the boundary of the MD domains. Since this paper focuses on the implementation of continuum conditions to MD simulations for PWC, the test cases are one-dimensional continuum simulations which use three dimensional molecular simulations for computing boundary conditions and viscosity. For this setup, the PWC scheme must distinguish between boundary cells and general cells away from the boundary. Both cases are shown schematically in Fig. 1. In the general case, the MD simulations are constrained by two sides (upper and lower). On both sides, the continuum state is imposed within a small region. The two other dimensions are periodic. When the lower side is bounded by a wall, this is modelled by MD. 
Enforcing the continuum constraints requires to alter the properties of the atoms inside the constrained region to match the continuum velocity, $\boldsymbol{u}_{\text {con }}$, and temperature, $T_{c o n}$. Additionally, the local continuum pressure, $P_{\text {con }}$, must be applied normal on the outer surface of the constrained region in order to keep the atoms within the molecular domain and to subject the molecular system to the correct pressure.

The velocity and temperature conditions can be imposed by two alternative methods. The first one is based on periodic rescaling of the atomic velocities [4, 10,22-26], and the second one on a periodic re-sampling from a velocity distribution functions, such as the MaxwellBoltzmann [15,27, 28] or the Chapman-Enskog [29-34] distribution.

\subsection{Rescaling techniques}

The average velocity of particles in the constrained region $R_{c t r}$ must correspond to the continuum velocity $\boldsymbol{u}_{\text {con }}$ :

$$
\frac{1}{M_{c t r}} \sum_{i \in R_{c t r}} m_{i} \boldsymbol{u}_{i}=\boldsymbol{u}_{c o n},
$$

where $M_{c t r}=\sum m_{i}, i \in R_{c t r}$ is the total mass of particles inside the constrained region. In order to satisfy Eq. (3) velocities, $\boldsymbol{u}_{i}$ of the atoms inside the constrained region are periodically replaced by $\boldsymbol{u}_{i}^{\prime}$, which is calculated by

$$
\boldsymbol{u}_{i}^{\prime}=\boldsymbol{u}_{i}-\frac{1}{M_{c t r}} \sum_{i \in R_{c t r}} m_{i} \boldsymbol{u}_{i}+\boldsymbol{u}_{\text {con }} .
$$

Hybrid methods usually apply the normal pressure through external forces $[9,22]$. The disadvantage of using an external force is that inserts/removes energy depending on the velocity of the atoms onto which the force is applied to. This results in oscillations in the molecular system $[6,16]$. The oscillations can be significantly reduced by using the velocity reversing scheme [6]. According to this, the pressure, $P_{c o n}$, is applied by reversing the velocity vector of atoms that move in the opposite direction of the pressure force. If the outer surface of the constrained region is normal to a dimension $\alpha$, then an atom $i$ is reversed by changing the sign of the respective velocity component: $\boldsymbol{v}_{i, \alpha}^{\prime}=-\boldsymbol{v}_{i, \alpha}^{\prime}$. For each reversed atom, $i$, a momentum $p_{i}=2 m_{i} \boldsymbol{v}_{i, \alpha}^{\prime}$ is applied. To apply a pressure of $P_{\text {con }}$ at each MD time step, the algorithm continues to reverse atoms until the transferred momentum equals the required momentum transfer due to the pressure:

$$
\sum_{i} 2 m_{i} \boldsymbol{v}_{i}^{\prime}=P_{c o n} \Delta t A_{c t r}
$$

where the sum is over the reversed atoms, $\Delta t$ is the size of the time step and $A_{c t r}$ is the surface area of the constrained region. The main advantages of the velocity reversing scheme are its simplicity, robustness, and the absence of any artifacts due to transfer of energy [6].

The continuum temperature is applied to the microscopic system through an energy transfer scheme [6]. The main idea is to add or remove energy from the microscopic system in order to match the macroscopic temperature without modifying the particles' mean velocity. The energy transfer is performed independently for each dimension and is achieved through scaling the velocity vectors of the atoms as follows:

$$
\boldsymbol{u}_{i}^{\prime}=\boldsymbol{u}_{i} f+c .
$$

The scaling factor, $f$, is calculated by

$$
f=\left(1+\frac{3 N_{c t r} k_{B} T_{c o n}}{2 E_{k, i n t}}\right),
$$


where $N_{c t r}$ is the number of atoms in the constrained regions, $E_{k, i_{n} t_{a}}$ is the internal kinetic energy of these atoms, $k_{B}$ denotes the Boltzmann's constant and $T_{c o n}$ is the target energy. The internal kinetic energy is given by

$$
E_{k, i n t}=\sum_{i \in R_{c t r}} \frac{1}{2} m_{i}\left(\boldsymbol{u}_{i}-\overline{\boldsymbol{u}}\right)^{2},
$$

with $\overline{\boldsymbol{u}}$ being the mean velocity component of the constrained atoms that is calculated by $\overline{\boldsymbol{u}}=$ $\left(1 / M_{c t r}\right) \sum_{i \in R_{c t r}} m_{i} \boldsymbol{u}_{i}$. The factor $\boldsymbol{c}$ is given by

$$
c=\bar{u}(1-f)
$$

and ensures that no momentum is transferred along with the energy.

\subsection{Resampling techniques}

The second BCT method utilises velocity distribution functions. For the scope of this study the atomistic velocities are periodically sampled either using the Maxwell-Boltzmann or the Chapman-Enskog distribution. Resampling has been previously applied by other authors in relation to the moving contact line problem [15,27].

The Maxwell-Boltzmann velocity distribution is the natural velocity distribution of an atomic or molecular system in an equilibrium state [35]. It defines the probability of the one-dimensional velocity components of an atom assuming a specific value, based on a temperature $T$ and the atom mass $m$.

For the Maxwell-Boltzmann distribution the probability density $f(\boldsymbol{C})$ of the thermal velocity $\boldsymbol{C}=\boldsymbol{u} /\left(2 k_{B} T / m\right)^{1 / 2}$ is given by

$$
f(\boldsymbol{C})=\frac{1}{\pi^{3 / 2}} \exp (-\boldsymbol{C}) .
$$

Each particle in the upper region is assigned a velocity $\boldsymbol{u}=\boldsymbol{u}_{\text {continuum }}+\boldsymbol{u}_{\text {maxwell }}$, where $\mathbf{u}_{\text {maxwell }}$ is the velocity of the Maxwellian distribution and $\boldsymbol{u}_{\text {continuum }}$ is the macroscopic velocity. The assigned atomistic velocities in the constrained region are then defined as

$$
u_{i a}=u_{a}^{c o n}+\sqrt{\frac{k_{B} T^{c o n}}{m_{i}}} \cdot \psi
$$

where $\psi$ denotes a Gaussian distributed number $N(0,1)$ and $u_{a}^{c o n}$ is the $a$ th component of the continuum velocity.

In order to ensure that every particle remains inside the molecular domain a reflective plane is placed at the upper boundary of the constrained region. This is simpler than the velocity reversing scheme, but can only be applied to incompressible flows because the normal pressure is a result of the reflected atoms.

For non-equilibrium situations the Chapman-Enskog distribution is a better model and, therefore, its application for sampling the atomic velocities has also been investigated. It has been used primarily in hybrid simulations of dilute gases that employ geometrical decomposition and state coupling [30-34].

The Chapman-Enskog distribution is a perturbed Maxwell-Boltzmann distribution [29] with probability density given by

$$
f(\boldsymbol{C})=\Gamma(\boldsymbol{C}) \pi^{-3 / 2} \exp \left(-\boldsymbol{C}^{2}\right),
$$


where $\Gamma(\boldsymbol{C})$ is the perturbation term given by

$$
\begin{aligned}
\Gamma(\boldsymbol{C})= & 1+\left(q_{x} u_{x}+q_{y} u_{y}+q_{z} u_{z}\right)\left(\frac{2}{5} \boldsymbol{C}^{2}-1\right)- \\
& 2\left(\tau_{x, y} C_{x} C_{y}+\tau_{x, z} C_{x} C_{z}+\tau_{y, z} C_{y} C_{z}\right)-\tau_{x, x}\left(C_{x}^{2}-C_{z}^{2}\right)- \\
& \tau_{y, y}\left(C_{y}^{2}-C_{z}^{2}\right),
\end{aligned}
$$

where $q_{a}$ and $\tau_{a, b},(a, b=x, y, z)$ denote the dimensionless heat flux and stress tensor, respectively. The atomistic velocities for the current distribution are sampled from Eq. (12) through the implementation of an acceptance-rejection random velocity generator described in Ref. 29.

\section{RESULTS AND DISCUSSION}

\subsection{Liquid flow for the boundary problem}

In this section the results of BCT schemes for liquid and gas flow and heat transfer problems are presented. The size of the molecular domain was $20 \sigma, 50 \sigma$ and $20 \sigma$ in $\mathrm{x}$, y and $\mathrm{z}$ directions, respectively. Periodic boundary conditions were applied in the $x$ and $z$ directions. Three regions were assigned: the wall at the bottom, the BCT region at top of the domain and the flow region in between.

The wall was modelled by two planes of a face-centred cubic (fcc) lattice, where the wall molecules were allowed to vibrate around their lattice sites by a harmonic spring with stiffness $k=50 \epsilon / \sigma^{2}$. Their velocities were rescaled to the wall temperature $T_{\text {wall }}=1.0 \epsilon / k_{B}$. The density of the wall atoms was $\rho_{\text {wall }}=1.0 \mathrm{~m}^{-3}$ and their mass was equal to that of the fluid atoms. Here, the wall properties do not correspond to any specific solid material, but represent a solid wall with no slip boundary condition. Similar models for solid walls have been used in previous studies $[27,36,37]$. The BCT region was located at $y>45 \sigma$ and the flow region at $1.5 \sigma<y<45 \sigma$. The total number of atoms was 18,820, of which 676 formed the wall and the remaining 18, 144 the fluid within the flow and BCT regions. The fluid density was $\rho_{\text {fluid }}=0.8 \mathrm{~m}^{-3}$ and the simulation time step was $\Delta t_{M D}=0.001 \tau$. Each simulation was run for $2 \times 10^{6}$ time steps and the calculated quantities were averaged over the last $2 \cdot 10^{5}$ time steps.

Initially, the rescaling technique and the one based on the Maxwell-Boltzmann distribution have been tested for a stationary heat transfer problem with continuum conditions $T_{\text {con }}=$ $1.5 \epsilon / k_{B}$ and $u_{c o n, x}=0 \sigma / \tau$ on the upper constrained region. The Chapman-Enskog distribution was utilised when the results obtained from the Maxwell-Boltzmann were not physically correct. A typical example is the application of the Maxwell-Boltzmann distribution in the dilute gases.

In the second test case the boundary conditions were $T_{\text {con }}=1.0 \epsilon / k_{B}$ and $u_{\text {con,x }}=$ $1.0 \sigma / \tau$. The temperature of the constrained region was equal to the wall temperature and, thus, the temperature was expected to remain nearly constant throughout the molecular domain. The results from both BCT methods are in good agreement with the theory as shown in Fig. 3. A linear velocity profile was obtained and the temperature remained constant and equal to $1.0 \epsilon / k_{B}$.

It was found that for the rescaling technique the size of the BCT region can influence the consistency of velocity values with the macroscopic conditions. In Eq. 1 the atomistic velocities are rescaled to a new mean velocity equal to the continuum constraint. In the current test case this results in an underestimated velocity in the lower boundary of the rescaled region (Fig. 4). The inconsistency between the macroscopic and microscopic states introduces inaccuracies in the simulation procedure. To address this issue, in the current test cases the BCT region has been further divided to four bins, with height $2.5 \sigma$ each. 


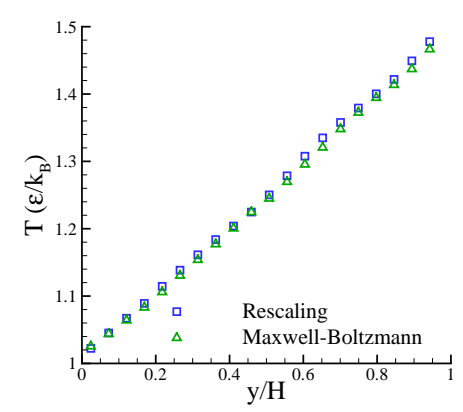

(a)

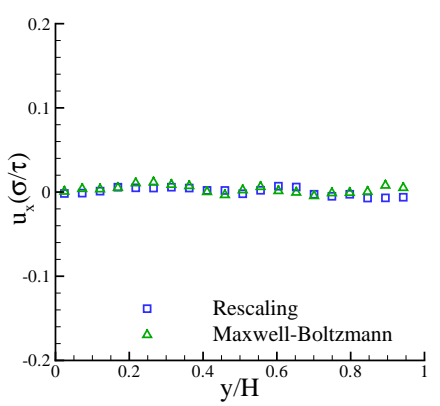

(b)

Fig. 2. Temperature profile for the Couette flow with $u_{x-c o n}=0 \sigma / \tau$ and $T_{\text {con }}=1.5 \mathrm{\epsilon} / k_{B}$.

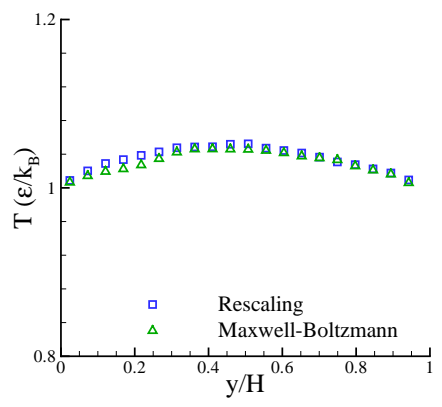

(a)

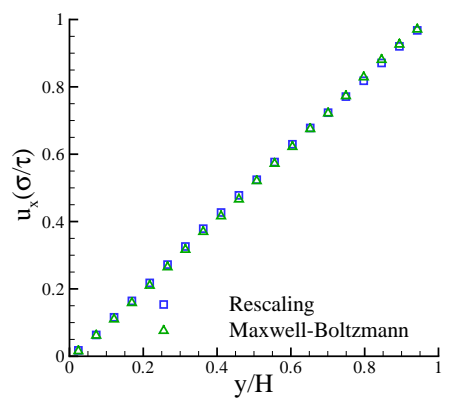

(b)

Fig. 3. Velocity and temperature profiles for the Couette flow with $u_{x-c o n}=1.0 \sigma / \tau$ and $T_{\text {con }}=1.0 \epsilon / k_{B}$.

For the third test case, the continuum conditions in the constrained region were $T_{\text {con }}=$ $1.2 \epsilon / k_{B}$ and $u_{c o n, x}=1.0 \sigma / \tau$. Both BCT methods provide similar results. Figure 5 shows the linear and parabolic profiles obtained for the velocity and temperature, respectively.

\subsection{Liquid flow for the general problem}

This is essentially a Couette flow problem with the molecular domain being constrained to continuum values on two opposite sides as illustrated in Fig. 1 (left). The size of the molecular domain was $10 \sigma, 30 \sigma$ and $10 \sigma$ in the $\mathrm{x}, \mathrm{y}$ and $\mathrm{z}$ dimensions, respectively. The molecular domain was subdivided into three regions: the upper and lower BCT regions and the flow region in between. Periodic boundary conditions were applied in the $x$ and $z$ dimensions. The lower constrained region was located at $y<5 \sigma$, the flow region at $5<y<25$ with height $H=20 \sigma$ and the upper constrained region at $y>25 \sigma$. The simulations were performed with a fluid density $\rho_{\text {fluid }}=0.8 \mathrm{m \sigma}^{-3}$, which resulted in a total number of 2, 592 particles. Using a time step $\Delta t_{M D}=0.001 \tau$, the simulations were run for $2 \times 10^{6}$ time steps. The temperature and velocity measurements were averaged over the last $2 \times 10^{5}$ time steps.

Three simulations were performed with different type of continuum conditions. In the first one, the temperature and velocity applied to the upper and lower boundaries were $u_{c o n, x}^{u p p p e r}=$ $0 \sigma / \tau, T_{\text {con }}^{\text {upper }}=1.5 \epsilon / k_{B}$ and $u_{\text {con }, x}^{\text {lower }}=0 \sigma / \tau, T_{\text {con }}^{\text {lower }}=1.0 \epsilon / k_{B}$ respectively. This setup corresponds to a pure heat transfer problem. Figure 6 shows the temperature and velocity 


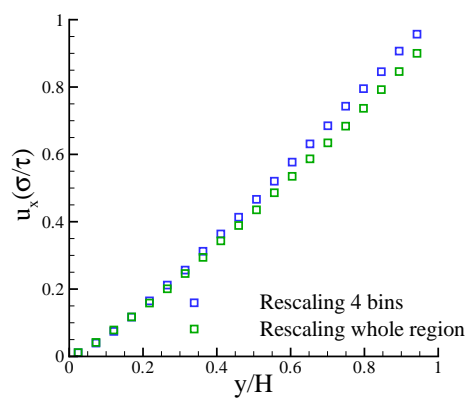

Fig. 4. Velocity profiles obtained with velocity constraints applied to the whole constrained region as well as the constrained region divided into subdomains.

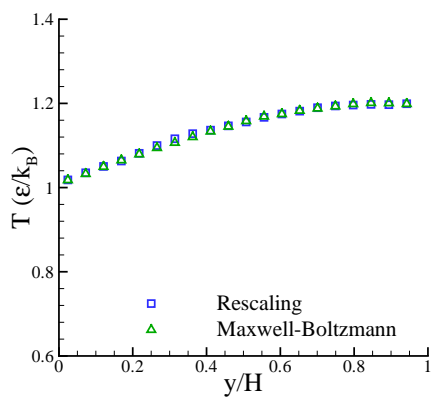

(a)

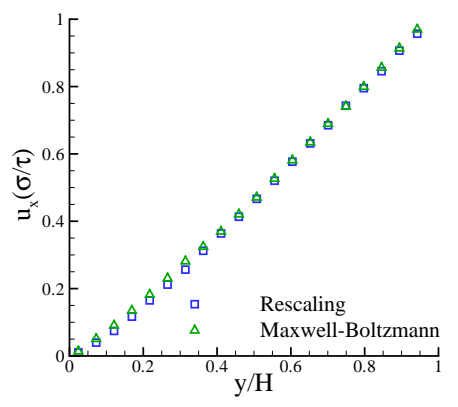

(b)

Fig. 5. Velocity and temperature profiles for the Couette flow with $u_{x-\text { con }}=1.0 \sigma / \tau$ and $T_{\text {con }}=1.2 \epsilon / k_{B}$.

profiles. As expected, the temperature profile obtained using both BCT methods is linear and the profile of the $x$ component of the velocity across the $y$ direction of the domain remains equal to zero. Furthermore, similar temperature profiles are obtained from both techniques. Small deviations are within the margin of statistical error due to the size of the MD domain and constrained region, as well as the time averaging procedure.

In the second simulation, the boundary conditions at the upper and lower boundaries were $u_{\text {con }, x}^{\text {upper }}=1.5 \sigma / \tau, T_{\text {con }}^{\text {upper }}=1.0 \epsilon / k_{B}$ and $u_{\text {con }, x}^{\text {lower }}=0.8 \sigma / \tau, T_{\text {con }}^{\text {lower }}=1.0 \epsilon / k_{B}$, respectively. The velocity and temperature profiles obtained from both BCT methods are similar as shown in Fig. 7.

For the third simulation, the applied boundary conditions were $u_{\text {con }, x}^{u p p p e r}=1.5 \sigma / \tau, T_{\text {con }}^{u p p e r}=$ $1.2 \epsilon / k_{B}$ and $u_{\text {coner } x}^{\text {lower }}=0.8 \sigma / \tau, T_{\text {con }}^{\text {lower }}=1.0 \epsilon / k_{B}$ at the lower and upper boundaries respectively. Results are shown in Fig. 8. The temperature profile is parabolic due to the heat generated by viscous dissipation and its conduction towards the boundaries [4]. Small deviations in the temperature profile are associated with statistical errors and the frequency with which the atomistic velocities are sampled from the velocity distribution function. The frequency with which the continuum constraints are applied is an important factor for the resampling method. A high frequency - in the extreme case, where resampling is performed at every time step - leads to a situation where the atoms are basically trapped inside the BCT region, because their velocities are continuously resampled and, therefore, change direction so that these atoms are almost 


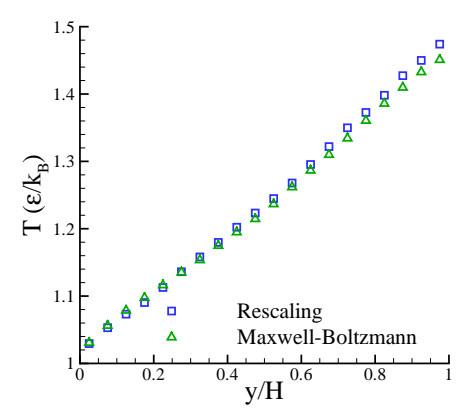

(a)

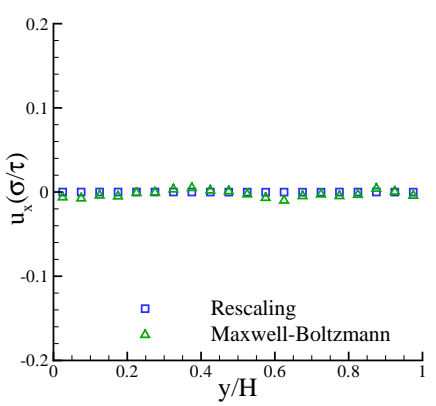

(b)

Fig. 6. Velocity and temperature profiles for the Couette flow with upper boundary conditions $u_{x-\text { con }}^{\text {upper }}=0 \sigma / \tau, T_{\text {con }}^{\text {upper }}=1.5 \epsilon / k_{B}$ and lower boundary conditions $u_{x-\text { con }}^{\text {lower }}=0 \sigma / \tau$, $T_{\text {con }}^{\text {lower }}=1.0 \epsilon / k_{B}$.

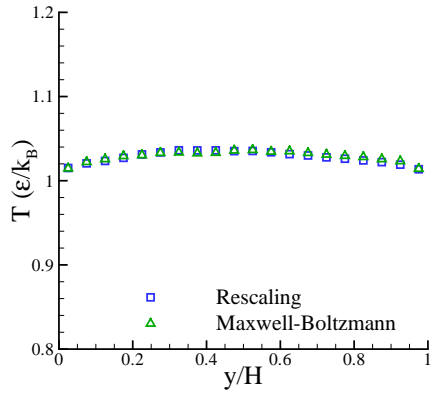

(a)

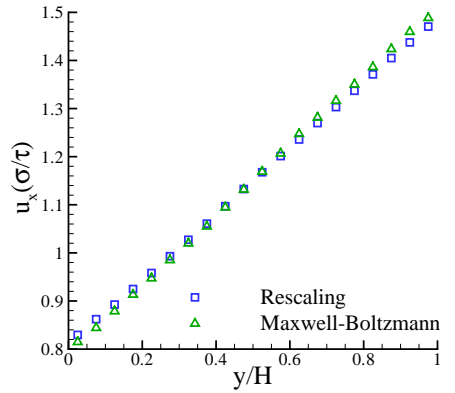

(b)

Fig. 7. Velocity and temperature profiles for the Couette flow with upper boundary conditions $u_{x-\text { con }}^{\text {upper }}=1.5 \sigma / \tau, T_{\text {con }}^{\text {upper }}=1.0 \epsilon / k_{B}$ and lower boundary conditions $u_{x-\text { con }}^{\text {lower }}=0.8 \sigma / \tau$, $T_{\text {con }}^{\text {lower }}=1.0 \epsilon / k_{B}$.

stationary. This effect becomes more apparent in the case of large BCT regions. On the other hand, one should be careful not to choose a too large resampling frequency that will not match the prescribed continuum state.

\subsection{Gas flows}

The second set of test cases aims to test the applicability of BCT methods to gas flows. The performed simulations were restricted to boundary node problems only. The size of the molecular domain was $200 \sigma, 120 \sigma$ and $200 \sigma$ in the $\mathrm{x}$, y and $\mathrm{z}$ directions, respectively, and similar to the liquid flows the domain was divided into two subregions; the flow region was located at $y<100 \sigma$ and the BCT region at $100 \sigma<y<120 \sigma$. At the bottom of the molecular domain a stochastic thermal wall was imposed. A stochastic thermal wall is similar to a reflective wall but corrects or resamples the velocity vector of the reflected atom depending on the transferred thermal energy to or from the wall. Such walls have been extensively used for gas flow simulations [38-41]. In the current study the following rules have been implemented by re-setting the 


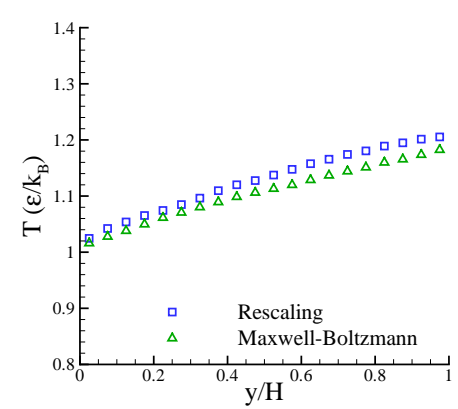

(a)

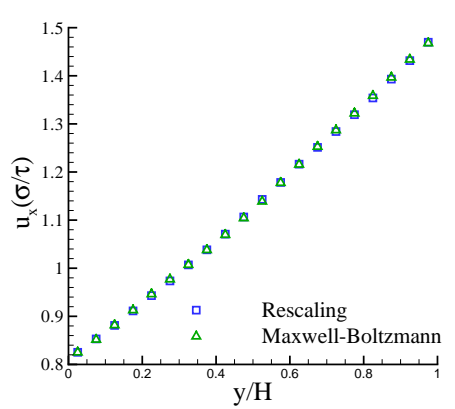

(b)

Fig. 8. Velocity and temperature profiles for the Couette flow with upper boundary conditions $u_{x-\text { con }}^{\text {upper }}=1.5 \sigma / \tau, T_{\text {con }}^{\text {upper }}=1.2 \epsilon / k_{B}$ and lower boundary conditions $u_{x-\text { con }}^{\text {lower }}=0.8 \sigma / \tau$, $T_{\text {coner }}^{\text {lower }}=1.0 \epsilon / k_{B}$.

velocities of each atom striking the wall:

$$
\begin{gathered}
v_{x}=\sqrt{\frac{k_{B} T_{\text {wall }}}{m}} \cdot \psi \\
v_{y}= \pm \sqrt{-\frac{2 k_{B} T_{\text {wall }}}{m} \cdot \ln \psi_{1}} \\
v_{z}=\sqrt{\frac{k_{B} T_{\text {wall }}}{m}} \cdot \psi^{\prime}
\end{gathered}
$$

where $T_{\text {wall }}$ is the wall temperature; $m$ is the atom's mass; $\psi$ and $\psi^{\prime}$ are Gaussian distributed random numbers, $N(0,1)$; and $\psi_{1}$ is a uniformly distributed random number in $U(0,1)$. Hence, in the adopted model the components of velocity which are parallel to the wall are sampled from a Maxwellian distribution

$$
f\left(v_{\alpha}\right)=\sqrt{\frac{m}{2 \pi k_{B} T_{\text {wall }}}} \exp \left(\frac{-m v_{\alpha}^{2}}{2 k_{B} T_{\text {wall }}}\right),
$$

where $\alpha$ is the corresponding direction and the normal to the wall velocity component, $v_{\beta}$, is sampled from a Rayleigh distribution given by

$$
f\left(v_{\beta}\right)=\frac{m}{k_{B} T_{\text {wall }}}\left|v_{\beta}\right| \exp \left(\frac{-m v_{\beta}^{2}}{2 k_{B} T_{\text {wall }}}\right) .
$$

The \pm in Eq. (14) corresponds the upper and lower walls, respectively. The + sign is used at the lower wall in order to force the particle to re-enter the simulation box. If the wall is placed at the upper boundary, the - sign is used.

The first test case concerns simulations where the BCT is enforced through a MaxwellBoltzmann distribution. The simulations have been performed for different values of gas density with continuum constraints $u_{c o n, x}=1.0 \sigma / \tau$ and $T_{c o n}=1.0 \epsilon / k_{B}$ and wall temperature $T_{\text {wall }}=1.0 \mathrm{\epsilon} / k_{B}$. Three values of density were simulated $\rho=0.02 \mathrm{~m}^{-3}, \rho=0.04 \mathrm{~m}^{-3}$ and $\rho=0.08 m \sigma^{-3}$ resulting in the generation of 10,240, 20, 000 and 40, 316 atoms, respectively. The time step used in the MD simulations was $\Delta t_{M D}=0.001 \tau$ and each simulation was run for $8 \times 10^{6}$ time steps. The macroscopic quantities were averaged over the last $2 \times 10^{6}$ time steps.

For low-density gas flows, slip at the boundary is expected, whose magnitude is related to the Knudsen number. High Knudsen numbers result in increased slip [36,39]. The Knudsen 

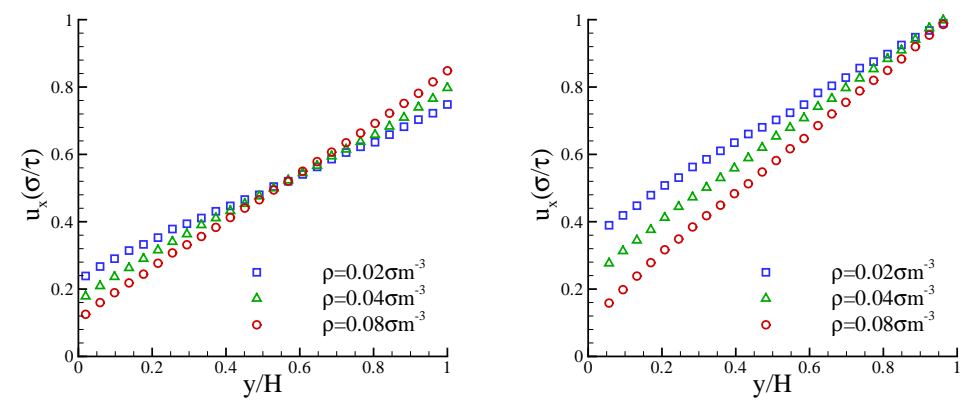

(a) Maxwell-Boltzmann based

BCT (b) Chapman-Enskog based BCT method method

Fig. 9. Velocity profiles obtained with Maxwell-Boltzmann and Chapman-Enskog distributions, respectively, for different gas densities.

number is calculated by [40]

$$
K n=\frac{\lambda}{L}=\frac{1}{\sqrt{2} \pi \rho \sigma^{2} L},
$$

where $\lambda$ is the mean free path of the gas, $\rho$ is the number density and $L$ is the characteristic length. Equation (17) means that low density results in higher Knudsen numbers and, consequently, higher magnitudes of the slip velocity. Figure 9(a) shows the velocity profiles obtained from the gas flow using the Maxwell-Boltzmann distribution based BCT scheme for the three densities. As expected, higher slip velocities near the wall are obtained for lower density values. However, large deviations are observed between the applied velocity constraints and the actual velocity in the upper boundary of the flow region. This is because of an additional slip velocity generated between the flow and BCT regions due to the application of the Maxwell-Boltzmann distribution. Note that lower gas density results in higher deviation between the actual and applied velocity $[15,33]$. To circumvent the unphysical slip at the constrained region, the same simulations have been performed with Maxwell-Boltzmann distribution replaced by the Chapman-Enskog distribution. Figure 9(b) shows velocity profiles obtained with the Chapman-Enskog distribution. Application of this distribution eliminates artificial slip phenomena between the flow and BCT regions.

For the last test case, the rescaling based technique and the method based on resampling the Chapman-Enkog's distribution are utilised for gas flow simulations in the same domain with the previous gas simulations, with density $\rho=0.05 \mathrm{mo}^{-3}$, and continuum constraints $u_{\text {con }, x}=1.0 \sigma / \tau$ and $T_{\text {con }}=1.0 \epsilon / k_{B}$ and wall temperature $T_{\text {wall }}=1.0 \epsilon / k_{B}$. In the simulations, 25, 168 particles have been generated, the MD time step was $\Delta t_{M D}=0.001 \tau$, each simulation was run for $8 \times 10^{6}$ time steps and the calculated macroscopic quantities were averaged over the last $2 \times 10^{6}$ time steps.

MD simulations of a larger system have been performed to verify the validity of the results. An MD domain of $200 \sigma$ in each direction was selected, comprising a total number of 42,592 particles. The density was $\rho=0.05 \mathrm{~m}^{-3}$ and the time step was $0.001 \tau$. The simulations were performed for $8 \times 10^{6}$ time steps and the calculated quantities were averaged over the last $2 \times 10^{6}$ time steps. Two stochastic thermal walls were placed at the upper and lower boundaries of the simulation domain with conditions chosen as $u_{\text {wall, } x}^{\text {upper }}=2.0 \sigma / \tau, T_{\text {wall }}^{\text {upper }}=0.8 \epsilon / k_{B}$ for the upper wall and $u_{\text {wall }, x}^{\text {lower }}=0 \sigma / \tau, T_{\text {wall }}^{\text {lower }}=1.0 \epsilon / k_{B}$ for the lower wall, respectively.

Figure 10(a) shows that results obtained from both BCT methods are in excellent agreement with the large MD simulation. Figure 10(b) shows the velocity distributions for continuum 


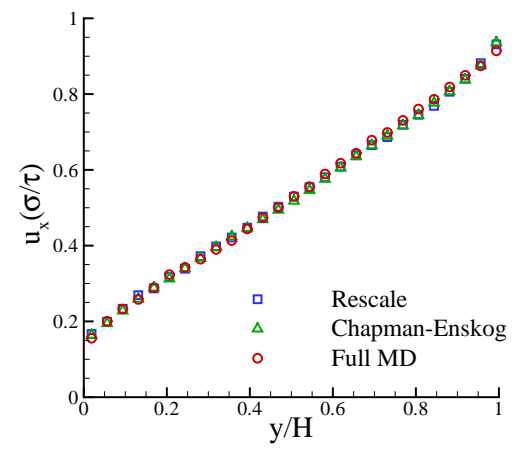

(a) $u_{x-c o n}=1 \sigma / \tau$

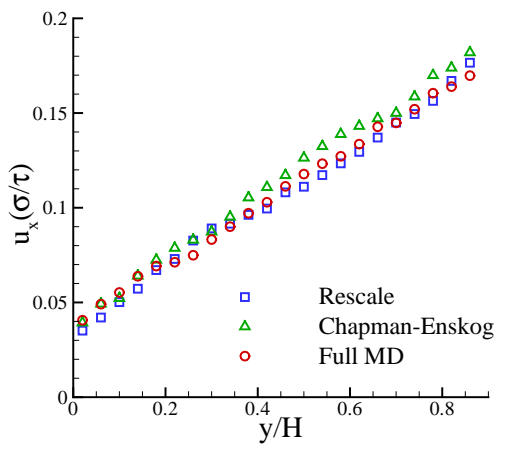

(b) $u_{x-c o n}=0.2 \sigma / \tau$

Fig. 10. Velocity profiles for gas with $\rho=0.05 \mathrm{~m} \cdot \sigma^{-3}$ obtained from the rescaling BCT method, the BCT method based on the Chapman-Enskog distribution and the full MD simulation.

velocity $u_{c o n, x}=0.2 \sigma / \tau=25 \mathrm{~m} / \mathrm{s}$.

\section{CONCLUSIONS}

An investigation of different mesoscale approaches for coupling macroscopic and microscopic simulations was presented. Two BCT methods of constraining the molecular domain to the continuum state have been examined, one based on rescaling the atoms' velocities and the other one is based on velocity sampling through a distribution function.

The rescaling BCT method was implemented in conjunction with a velocity reversing scheme with regards to the macroscopic pressure. Applying the correct value of pressure and minimising any associated artifacts is crucial for the performance of any hybrid scheme. Inconsistencies in the pressure can shrink the simulation domain or even make particles drift away. This can generate errors and instabilities in the hybrid procedure. Simulations performed with the rescaling BCT method show that it can be successfully applied to both liquid and gas flows. The size of the regions where the velocity constraints are applied has to be selected carefully in order to obtain consistent velocities with the continuum state.

For the second BCT method, Maxwell-Boltzmann and Chapman-Enskog distribution functions were examined. The former has been used in liquid simulations and the results were found in good agreement with the rescaling BCT method. The size of the constrained domain, the way that the domain is terminated and the sampling frequency may have significant impact on the results when the Maxwell-Boltzmann distribution is used. Inadequate sampling frequency can lead to unrealistic effects, such as trapping of particles in the constrained region, or deviations between the macroscopic and microscopic velocities. Selection criteria of these parameters depends on the problem in question and cannot be defined explicitly. The application of the Maxwell-Boltzmann distribution to gas flows leads to discrepancies between the desired and the actual applied velocity. This discrepancy can be corrected by applying the Chapman-Enskog distribution. The results obtained are then consistent with the rescaling-based BCT method and larger MD simulations.

The selection of BCT method is not a trivial issue. It depends on the specific simulated problem and a number of other parameters such as the accuracy requirements and the available computational resource. The main advantage of the rescaling method is its generic nature and broad range of applicability. However, it is less computationally efficient compared to the velocity distribution function method. 


\section{Acknowledgments}

The financial support from EPSRC, MoD and AWE through the grant EP/D051940-JGS 607, as well as from the European Commission under the 6th Framework Program (Project: DINAMICS, NMP4-CT-2007-026804), is greatly acknowledged.

\section{References}

[1] G. Whitesides, "The origins and the future of microfluidics," Nature 442, 368-373 (2006) [doi:10.1038/nature05058].

[2] A. E. Kamholz, B. H. Weigl, B. A. Finlayson, and P. Yager, "Quantitative analysis of molecular interaction in a microfluidic channel: The t-sensor," Anal. Chem. 71(23), 53405347 (1999) [doi:10.1021/ac990504j].

[3] M. Gad-El-Hak, "Gas and liquid transport at the microscale," Heat Tran. Eng. 27(4), 13 29 (2006) [doi:10.1080/01457630500522305].

[4] J. Liu, S. Chen, X. Nie, and M. O. Robbins, "A continuum-atomistic simulation of heat transfer in micro- and nano-flows," J. Comput. Phys. 227(1), 279-291 (2007) [doi:10.1016/j.jcp.2007.07.014].

[5] S. O'connell and P. Thompson, "Molecular dynamics-continuum hybrid computations: A tool for studying complex fluid flows," Phys. Rev. E 52(6), R5792-R5795 (1995) [doi:10.1103/PhysRevE.52.R5792].

[6] M. Kalweit and D. Drikakis, "Coupling strategies for hybrid molecularcontinuum simulation methods," J. Mech. Eng. Sci. 222, 797-806(10) (2008) [doi:10.1243/09544062JMES716].

[7] E. G. Flekkoy, G. Wagner, and J. Feder, "Hybrid model for combined particle and continuum dynamics," Europhys. Lett. 52(3), 271-276 (2000) [doi:10.1209/epl/i2000-00434-8].

[8] E. G. Flekkoy, R. Delgado-Buscalioni, and P. V. Coveney, "Flux boundary conditions in particle simulations," Phys. Rev. E 72(2), 1-9 (2005) [doi:10.1103/PhysRevE.72.026703].

[9] T. Werder, J. H. Walther, and P. Koumoutsakos, "Hybrid atomistic-continuum method for the simulation of dense fluid flows," J. Comput. Phys. 205(1), 373-390 (2005) [doi:10.1016/j.jcp.2004.11.019].

[10] X. Nie, M. O. Robbins, and S. Chen, "Resolving singular forces in cavity flow: Multiscale modeling from atomic to millimeter scales," Phys. Rev. Lett. 96(13), 1-4 (2006) [doi:10.1103/PhysRevLett.96.134501].

[11] Y. Wang and G. He, "A dynamic coupling model for hybrid atomistic-continuum computations," Chem. Eng. Sci. 62(13), 3574-3579 (2007) [doi:10.1016/j.ces.2006.12.093].

[12] J. Li, D. Liao, and S. Yip, "Nearly exact solution for coupled continuum/md fluid simulation," J.Comp.-Aid. Mater. Des 6(2), 95-102 (1999) [doi:10.1023/A:1008731613675].

[13] N. Asproulis, M. Kalweit, and D. Drikakis, "A hybrid molecular continuum method using point wise coupling," in Sixth International Conference on Engineering Computational Technology, B. Topping and M. Papadrakakis, Eds., Civil-Comp Press (2008) [doi:10.4203/ccp.89.38].

[14] N. Asproulis and D. Drikakis, "Nanoscale materials modeling using neural networks," $J$. Comp. Theor. Nanosci. 6(3), 514-518 (2009) [doi:10.1166/jctn.2009.1062].

[15] N. G. Hadjiconstantinou and A. T. Patera, "Heterogeneous atomistic-continuum representations for dense fluid systems," Int. J. Mod. Phys. C 8(4), 967-976 (1997) [doi:10.1142/S0129183197000837].

[16] M. Kalweit and D. Drikakis, "Multiscale methods for micro/nano flows and materials," $J$. Comp. Theor. Nanosci. 5(9), 1923-1938 (2008) [doi:10.1166/jctn.2008.906].

[17] N. Asproulis, E. Shapiro, M. Kalweit, and D. Drikakis, "Multiscale modelling for flows and materials," in Cranfield Multi-Strand Conference, Cranfield, United Kingdom (2008). 
[18] N. G. Hadjiconstantinou, "Discussion of recent developments in hybrid atomisticcontinuum methods for multiscale hydrodynamics," Bull. Poli. Acad. Sci.: Tech. Sci. 53(4), 335-342 (2005) [doi:10.1615/IntJMultCompEng.v2.i2.20].

[19] G. Sutmann, Quantum Simulations of Complex Many-Body Systems: From Theory to Algorithms, Lecture Notes, vol. 10, ch. Classical molecular dynamics, 211-254. John von Neumann Institute for Computing, Juelich, NIC Series (2002).

[20] S. Plimpton, "Fast parallel algorithms for short-range molecular dynamics," J. Comput. Phys. 117(1), 1-19 (1995) [doi:10.1006/jcph.1995.1039].

[21] L. Verlet, "Computer 'experiments' on classical fluids. I. Thermodynamical properties of Lennard-Jones molecules," Phys. Rev. 159, 98-103 (1967) [doi:10.1103/PhysRev.159.98].

[22] X. B. Nie, S. Y. Chen, W. N. E, and M. O. Robbins, "A continuum and molecular dynamics hybrid method for micro- and nano-fluid flow," J. Fluid Mech. (500), 55-64 (2004) [doi:10.1017/S0022112003007225].

[23] R. Delgado-Buscalioni and P. Coveney, "Usher: An algorithm for particle insertion in dense fluids," J. Chem. Phys. 119, 978-987 (2003) [doi:10.1063/1.1579475].

[24] R. Delgado-Buscalioni and P. Coveney, "Continuum-particle hybrid coupling for mass, momentum and energy transfers," Phys. Rev. E 67, 046704 (2003) [doi:10.1103/PhysRevE.67.046704].

[25] R. Delgado-Buscalioni and P. Coveney, "Hybrid molecular-continuum fluid dynamics," Phil. Trans. R. Soc. Lond. A 362, 1639-1654 (2004).

[26] G. D. Fabritiis, R. Delgado-Buscalioni, and P. V. Coveney, "Multiscale modeling of liquids with molecular specificity," Phys. Rev. Lett. 97, 134501 (2006) [doi:10.1103/PhysRevLett.97.134501].

[27] N. G. Hadjiconstantinou, "Combining atomistic and continuum simulations of contact-line motion,” Phys. Rev. E 59, 2475 (1999) [doi:10.1103/PhysRevE.59.2475].

[28] W. Ren and E. Weinan, "Heterogeneous multiscale method for the modeling of complex fluids and micro-fluidics," J. Comput. Phys. 204(1), 1-26 (2005) [doi:10.1016/j.jcp.2004.10.001].

[29] A. L. Garcia and B. J. Alder, "Generation of the chapman-enskog distribution," J. Comput. Phys. 140(1), 66-70 (1998) [doi:10.1006/jcph.1998.5889].

[30] T. E. Schwartzentruber, L. C. Scalabrin, and I. D. Boyd, "A modular particle-continuum numerical method for hypersonic non-equilibrium gas flows," J. Comput. Phys. 225(1), 1159-1174 (2007) [doi:10.1016/j.jcp.2007.01.022].

[31] T. E. Schwartzentruber, L. C. Scalabrin, and I. D. Boyd, "Hybrid particle-continuum simulations of hypersonic flow over a hollow-cylinder-flare geometry," AIAA Journal 46(8) (2008) [doi: 10.2514/1.36681].

[32] T. E. Schwartzentruber, L. C. Scalabrin, and I. D. Boyd, "Hybrid particle-continuum simulations of nonequilibrium hypersonic blunt-body flowfields," J. Therm. Heat Tran. 22(1), 29-37 (2008) [doi:10.2514/1.30216].

[33] H. S. Wijesinghe, R. D. Hornung, A. L. Garcia, and N. G. Hadjiconstantinou, "Threedimensional hybrid continuum-atomistic simulations for multiscale hydrodynamics," $J$. Fluid Eng. 126(5), 768-777 (2004) [doi:10.1115/1.1792275].

[34] H. S. Wijesinghe and N. G. Hadjiconstantinou, "A hybrid atomistic-continuum formulation for unsteady, viscous, incompressible flows," CMES 5(6), 515-526 (2004).

[35] N. Bimalendu, Fundamentals of Classical and Statistical Thermodynamics, John Wiley, West Sussex, United Kingdom (2002).

[36] B. Y. Cao, "Non-maxwell slippage induced by surface roughness for microscale gas flow: A molecular dynamics simulation," Mol. Phys. 105(10), 1403-1410 (2007) [doi:10.1080/00268970701361322]. 
[37] T. M. Galea and P. Attard, "Molecular dynamics study of the effect of atomic roughness on the slip length at the fluid-solid boundary during shear flow," Langmuir 20(8), 3477-3482 (2004) [doi:10.1021/la035880k].

[38] A. Tenenbaum, "Local equilibrium in stationary states by molecular dynamics," Phys. Rev. A 28(5), 3132-3133 (1983) [doi:10.1103/PhysRevA.28.3132].

[39] R. Tehver, F. Toigo, J. Koplik, and J. R. Banavar, "Thermal walls in computer simulations," Phys. Rev. E 57(1),R17-R20 (1998) [doi:10.1103/PhysRevE.57.R17].

[40] D. K. Bhattacharya and G. C. Lie, "Nonequilibrium gas flow in the transition regime: A molecular-dynamics study," Phys. Rev. A 43(2), 761-767 (1991) [doi:10.1103/PhysRevA.43.761].

[41] J. L. Xu and Z. Q. Zhou, "Molecular dynamics simulation of liquid argon flow at platinum surfaces," Heat Mass Transf. 40(11), 859-869 (2004) [doi:10.1007/s00231-003-0483-3]. 